\title{
THIAMINE HYDROCHLORIDE AS EFFICIENT CATALYST FOR ONE-POT SYNTHESIS OF 14-ARYL -14H DIBENZO [a, j] XANTHENES UNDER GREENERY CONDITIONS
}

\author{
Uppalaiah Kusampally, Ramgopal Soma and Chinna Rajanna Kamatala* \\ Department of Chemistry, Osmania University, Hyderabad 500 007, Telangana, India \\ *E-mail : : kcrajannaou@ yahoo.com
}

\begin{abstract}
Thiamine hydrochloride (THC) is a biodegradable, readily available and inexpensive eco-friendly metal free biocatalyst, which is efficiently used in the condensation of 2-naphthol and aromatic aldehydes under thermal and microwave conditions without using toxic solvents. Developed methods herein afforded 14-aryl 14H-dibenzo [a, j] xanthenes in very good yields and offered several advantages such as shorter reaction time, comparable yield, neat practice, easy work-up and mild organo Lewis acid catalyst.

Keywords: Thiamine hydrochloride (THC), Biodegradable mild organo Lewis acid catalyst, Solvent Free and Micro wave condition, One-pot synthesis of 14-aryl 14H-dibenzo [a, j] xanthenes, High Yields, Easy workup.

(C) RASĀYAN. All rights reserved
\end{abstract}

\section{INTRODUCTION}

Benzoxanthenes fall under a special category of xanthenes, which are known to exhibit antiviral and antibacterial properties ${ }^{1-3}$. Some of the benzoxanthenes find their applications in the preparation of dyes ${ }^{3}$, fluorescent compounds ${ }^{4}$, photodynamic therapy, and also in laser technologies ${ }^{5-9}$. In a recent publication ${ }^{1}$ Ganeshet al revealed that benzoxanthenes can be prepared by the condensation of $\beta$-naphthol with varieties of aldehydes using different types of catalysts like mineral acids, Lewis acids, carboxylic acids, sulfonic acids, molecular iodine and ionic liquids, $\mathrm{NaHSO}_{4}$ and aluminum bisulfate. In addition to a helpful review of literature in this publication, Singh and coworkers ${ }^{1}$ developed an eco-friendly solventfree condensation method to obtain 14- aryl- or alkyl-14H-dibenzo [a, j] xanthenes using $\mathrm{P}_{2} \mathrm{O}_{5}$ or $\mathrm{InCl}_{3}$ as catalysts. For the synthesis of 14-aryl-14H-dibenzo [a, j] xanthene derivatives, Geng-Chen Li, has used aryl aldehydes, and freshly prepared expanded graphite as a catalyst ${ }^{10}$, while Hamid Reza Shaterian et al., reported the use of ferric hydrogen sulfate and aluminum hydrogen sulfate as catalysts ${ }^{11,12}$. Carbon tetra bromide $\left(\mathrm{CBr}_{4}\right)^{13}$, and polyvinyl poly pyrrolidine-supported boron trifluoride $\left(\mathrm{PVPP}-\mathrm{BF}_{3}\right)^{14}$ were the other important catalysts used for the synthesis of dibenzo xanthenes. FarhadShirini et al illustrated the synthesis of benzoxanthenes compounds through the condensation of 2-naphthol and 1,3 cyclohexanedione with and without succinimide $\mathrm{N}$ - sulfonic acid as catalyst ${ }^{15}$. In another MCR protocol, Javad Safaei-Ghomi and Mohammad $\mathrm{Ali}^{16}$ used recoverable AgI nano particles as a catalyst to achieve 14-aryl-14H-dibenzo [a, j] xanthenes via the reaction of 2-naphthol with different aldehydes. In another publication Davinder Prasad and fellow researchers used $p$-dodecylbenzesulfonic acid as a catalyst in MW assisted green synthesis of dibenzo [a,j] xanthenes to achieve good product yields ${ }^{17}$. As a matter of fact efforts several workers focused their attention on improving the greenery in the synthesis of 14-Aryl $-14 \mathrm{H}$ Dibenzo [a, j] xanthenes by using large varieties of catalysts such as, $[\mathrm{H}-\mathrm{NMP}]\left[\mathrm{HSO}_{4}\right]^{18},\left(\mathrm{SiO}_{2}-\mathrm{Pr}-\right.$ $\left.\mathrm{SO}_{3} \mathrm{H}\right)^{19}$, N-sulfonic acid poly(4-vinyl pyridinium) chloride (NSPVPC) ${ }^{20}$, 1-methyl-3-propane sulfonicimidazolium hydrosulfate ([MIMPS] $\left.\mathrm{HSO}_{4}\right)^{21}$ and many more ${ }^{22}$. Many of these protocols are conducted either under solvent-free conditions and/or under the MW irradiation; because MW assisted reactions to accelerate the reactions through bulk activation of molecules and give product yields ${ }^{23}$. Enthused by these factors, we want to explore readily available and inexpensive thiamine hydrochloride (THC) as a

Rasayan J. Chem., 12(1), 152-156(2019)

http://dx.doi.org/10.31788/RJC.2019.1215043

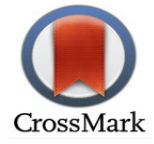


biodegradable and ecofriendly catalyst in the one-pot synthesis of 14-aryl-14H-dibenzo [a, j] xanthene derivatives using condensation of aromatic aldehydes with $\beta$-naphthol under solvent-free and MW conditions (Scheme-1).<smiles>[R]c1ccccc1C=O</smiles>

2

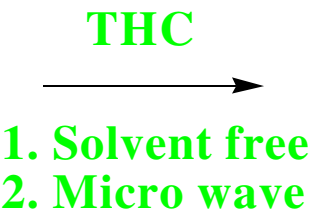

2. Micro wave<smiles>[R]c1ccccc1C1c2ccc3ccccc3c2Oc2cccc3cccc1c23</smiles>

3

Scheme-1: THC accelerated One-pot Synthesis of 14-Aryl-14H-Dibenzo [a, j] Xanthenes

\section{EXPERIMENTAL}

\section{Solvent Free Method for Synthesis of Xanthene Derivatives}

Round bottomed reaction flask containing $\beta$-naphthol $(2 \mathrm{mmol})$, benzaldehyde $(1 \mathrm{mmol})$ and THC (100 $\mathrm{mg}$ ) was placed in the mantle and refluxed at about $120{ }^{\circ} \mathrm{C}$. After the reaction is completed (as indicated by TLC), the reaction mixture was kept aside for cooling to room temperature. The reaction mixture was neutralized with $15 \% \mathrm{Na}_{2} \mathrm{CO}_{3}$; ethyl acetate was added to the flask to extract organic components. Finally, the resultant organic layer was run through the column containing silica gel (60-120 mesh size). Separated product was again recrystallized with $15 \%$ aqueous EtOH.

\section{Microwave Irradiation for Synthesis of Xanthene Derivatives}

After preparing the reaction mixture as mentioned in the preceding section, the reaction flask was placed in a laboratory microwave oven (BPL, 800T model) on a silica gel solid support and allowed to heat at $300 \mathrm{~W}$ for the appropriate time. After completion, the product was isolated and purified using the above workup.

\section{RESULTS AND DISCUSSION}

Initially, the reaction conditions are optimized using $\beta$-naphthol $(2 \mathrm{mmol})$, benzaldehyde $(1 \mathrm{mmol})$, and varied amounts of biodegradable thiamine hydrochloride (THC) catalyst in different reaction media, under also solvent-free conditions. Obtained results are presented in Table-1.

Table-1: Optimization of Catalyst Thiamine Hydrochloride (THC):

\begin{tabular}{c|c|c|c|c|c}
\hline S. No. & Reaction Media & THC $(\mathrm{mg})$ & Temp. $(0 \mathrm{C})$ & Time & 3a Yield (\%) \\
\hline 1 & Acetonitrile & 100 & Reflux & 4.0 hours & 20 \\
\hline 2 & Dichloro Ethane & 100 & Reflux & 4.0 hours & 35 \\
\hline 3 & Ethanol & 100 & Reflux & 3.0 hours & 60 \\
\hline 4 & Methanol & 100 & Reflux & 3.0 hours & 50 \\
\hline 5 & Chloroform & 100 & Reflux & 3.5 hours & 43 \\
\hline 6 & Water & 100 & Reflux & 5.0 hours & 12 \\
\hline 7 & None & 0 & 0 & 3.0 hours & 0 \\
\hline 8 & None & 40 & 120 & 3.0 hours & 53 \\
\hline 9 & None & 60 & 120 & 120 minutes & 66 \\
\hline 10 & None & 80 & 120 & 75 minutes & 79 \\
\hline 11 & None & 100 & 120 & 45 minutes & 92 \\
\hline 12 & None & 120 & 120 & 50 minutes & 92 \\
\hline 13 & None & 120 & MW assistance & $4-5$ min & 92 \\
\hline
\end{tabular}


RASĀYAN J. Chem.

Vol. 12 | No. 1 |152 - 156| January - March | 2019

Under solvent-free conditions and using $100 \mathrm{mg}$ THC the reaction afforded excellent product (3a) yield at $120^{\circ} \mathrm{C}$. This reaction required 45 minutes for completion in solvent-free conditions. But MW assisted reaction under otherwise similar conditions took only 4 to 5 and gave desirable yields. Under optimized conditions, a large array of aldehydes were used to get corresponding 14-aryl-14H-dibenzo [a, j] xanthene derivatives (3a-3p). Products were characterized by physical spectroscopic (mass, IR, NMR and C13NMR data). Reaction times and product yields are presented in table-2.The most plausible mechanism is proposed as shown in Scheme-2, which shows that THC acts as an acid catalyst to produce $\left(\mathrm{H}^{+}\right)$ions with internal rearrangement of THC salt. First step, proton is abstracted by the aryl aldehyde leads to the formation of aryl oxocarbenium ion intermediate (II), which in turn reacts with $\beta$-naphthol in a condensation step to generate naphthyl oxonium intermediate (III) by the elimination of water molecule as shown Scheme-1, which is further reacted with one more $\beta$-naphthol molecule to form target compound (IV) by the removal of $\mathrm{H}_{2} \mathrm{O}$ molecule. Total progress of the reaction parameters such as time and yields are tabulated in Table-2 for their respective derivatives.

Table -2: One-pot Synthesis of 14-aryl -14H Dibenzo [a, j] Xanthene Derivatives.

\begin{tabular}{|c|c|c|c|c|c|c|}
\hline \multirow[t]{2}{*}{ S. No. } & \multirow[t]{2}{*}{ Aldehydes } & \multirow{2}{*}{$\begin{array}{l}\text { Products } \\
\text { (3) }\end{array}$} & \multicolumn{2}{|c|}{${ }^{1}$ Solvent Free } & \multicolumn{2}{|c|}{${ }^{2}$ Micro Wave } \\
\hline & & & $\begin{array}{c}\text { R.T } \\
(\min )\end{array}$ & Yield (\%) & $\begin{array}{c}\text { R.T } \\
(\min )\end{array}$ & Yield (\%) \\
\hline 1 & $\mathrm{C}_{6} \mathrm{H}_{5}-\mathrm{CHO}$ & $3 a$ & 43 & 92 & 4 & 96 \\
\hline 2 & $2-\mathrm{NO}_{2}-\mathrm{C}_{6} \mathrm{H}_{4}-\mathrm{CHO}$ & $3 b$ & 45 & 86 & 5 & 92 \\
\hline 3 & 4-Et- $\mathrm{C}_{6} \mathrm{H}_{4}-\mathrm{CHO}$ & $3 c$ & 44 & 90 & 4 & 95 \\
\hline 4 & $4-\mathrm{Me}-\mathrm{C}_{6} \mathrm{H}_{4}-\mathrm{CHO}$ & $3 d$ & 42 & 92 & 4 & 96 \\
\hline 5 & $4-\mathrm{MeO}-\mathrm{C}_{6} \mathrm{H}_{4}-\mathrm{CHO}$ & $3 \mathrm{e}$ & 42 & 93 & 4 & 96 \\
\hline 6 & $4-\mathrm{OH}-\mathrm{C}_{6} \mathrm{H}_{4}-\mathrm{CHO}$ & $3 \mathrm{f}$ & 42 & 93 & 4 & 97 \\
\hline 7 & $2-\mathrm{Cl}-\mathrm{C}_{6} \mathrm{H}_{4}-\mathrm{CHO}$ & $3 g$ & 43 & 91 & 4 & 95 \\
\hline 8 & $4-\mathrm{Cl}-\mathrm{C}_{6} \mathrm{H}_{4}-\mathrm{CHO}$ & $3 \mathrm{~h}$ & 42 & 93 & 4 & 96 \\
\hline 9 & $\mathrm{CH}_{3}-\mathrm{CH}_{2}-\mathrm{CHO}$ & $3 \mathrm{i}$ & 44 & 90 & 5 & 95 \\
\hline 10 & $\mathrm{C}_{6} \mathrm{H}_{4}-\mathrm{CHO}$ & $3 \mathrm{j}$ & 45 & 90 & 5 & 95 \\
\hline 11 & $4-\mathrm{Br}-\mathrm{C}_{6} \mathrm{H}_{4}-\mathrm{CHO}$ & $3 \mathrm{k}$ & 44 & 92 & 5 & 96 \\
\hline 12 & $3-\mathrm{OH}-\mathrm{C}_{6} \mathrm{H}_{4}-\mathrm{CHO}$ & 31 & 43 & 92 & 4 & 96 \\
\hline 13 & $4-\mathrm{NO}_{2}-\mathrm{C}_{6} \mathrm{H}_{4}-\mathrm{CHO}$ & $3 \mathrm{~m}$ & 45 & 89 & 5 & 93 \\
\hline 14 & $3-\mathrm{MeO}-\mathrm{C}_{6} \mathrm{H}_{4}-\mathrm{CHO}$ & $3 n$ & 43 & 91 & 4 & 94 \\
\hline 15 & $4-\mathrm{CN}-\mathrm{C}_{6} \mathrm{H}_{4}-\mathrm{CHO}$ & 30 & 44 & 92 & 4 & 95 \\
\hline 16 & $3-\mathrm{F}-\mathrm{C}_{6} \mathrm{H}_{4}-\mathrm{CHO}$ & $3 p$ & 45 & 92 & 4 & 96 \\
\hline
\end{tabular}

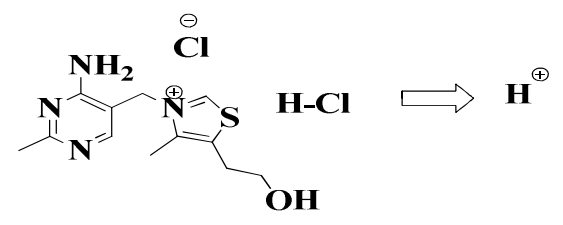

Thiamine Hydrochloride (THC)

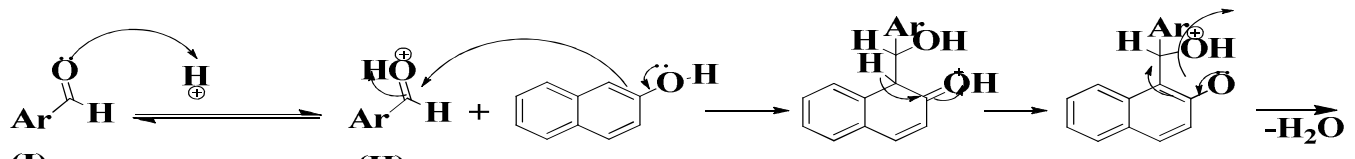

(I)

(II)

(III)

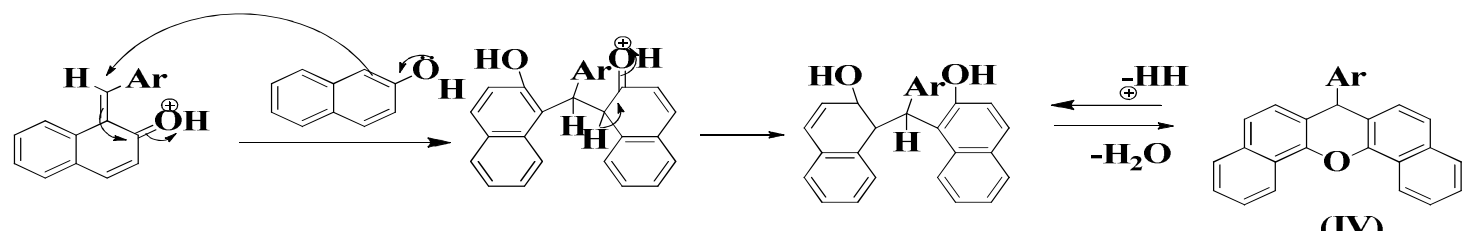

Scheme-2: Plausible Mechanism for the Formation of 14-Aryl -14H Dibenzo [a, j] Xanthenes 
In MW assisted reactions all the molecules acquire energy directly from the source without any time lapse from MW irradiation, which causes bulk activation of molecules. The fraction of activated molecules grows exponentially under MW irradiation when compared to the thermal reactions which occur due to thermal collisions. Due to this bulk activation of molecules reaction, times reduce enormously, accelerate the reactions and afford higher product yields, as explained by several workers ${ }^{23-28}$.

\section{Characterization of Products}

The spectral $\left({ }^{1} \mathrm{HNMR},{ }^{13} \mathrm{CNMR}\right.$, IR, and $\left.\mathrm{m} / \mathrm{z}\right)$ data of few representative compounds are as follows:

\section{4-Phenyl-14H-dibenzo [a,j] Xanthene (3a)}

White solid; MP. $184-186{ }^{\circ} \mathrm{C} ;{ }^{1} \mathrm{HNMR}\left(400 \mathrm{MHz}, \mathrm{CDCl}_{3}\right): \delta=6.48(\mathrm{~s}, 1 \mathrm{H}), 6.79(\mathrm{t}, J=8.4 \mathrm{~Hz}, 2 \mathrm{H}), 7.37-$ $7.46(\mathrm{~m}, 6 \mathrm{H}), 7.56(\mathrm{t}, J=8.0 \mathrm{~Hz}, 2 \mathrm{H}), 7.78(, J=8.4 \mathrm{~Hz}, 2 \mathrm{H}) ;{ }^{13} \mathrm{CNMR}\left(100 \mathrm{MHz}, \mathrm{CDCl}_{3}\right): \delta=38.2$, 117.4, 118.1, 122.5, 124.3, 126.4, 126.8, 128.3, 128.3, 128.8, 128.9, 131.1, 131.3, 148.8; IR $\left(\mathrm{KBr}, \mathrm{Cm}^{-1}\right)$ : $3075,1582,1444,1252,1081,966 ; \mathrm{m} / \mathrm{z}(\%)=358\left(\mathrm{M}^{+}\right), 281,268$.

\section{4-(2-Nitrophenyl)-14H-dibenzo [a, j] Xanthene (3b)}

Yellow solid; MP. $293{ }^{0} \mathrm{C}$; ${ }^{1} \mathrm{HNMR}\left(300 \mathrm{MHz}, \mathrm{CDCl}_{3}\right): \delta=6.45(\mathrm{~s}, 1 \mathrm{H}), 6.98-7.17(\mathrm{~m}, 3 \mathrm{H}), 7.40-7.85$ $(\mathrm{m}, 12 \mathrm{H}), 8.38(\mathrm{~d}, J=8.4 \mathrm{~Hz}, 2 \mathrm{H}) ;{ }^{13} \mathrm{CNMR}\left(75 \mathrm{MHz}, \mathrm{CDCl}_{3}\right): \delta 32.9,118.4,124.6,125.3,128.0,129.5$, 130.8, 132.6, 134.5, 141.3, 147.5, 149.8; IR ( $\left.\mathrm{KBr} \mathrm{Cm}^{-1}\right)$ : 3400, 3058, 1593, 1523, 1350, 1240, 1142, 810, 748; m/z: 403( $\left.\mathrm{M}^{+}\right), 281,268$.

14-(4-methylphenyl)-14H-dibenzo [a, j] Xanthene (3d)

Yellow Solid; MP. $224-226{ }^{0} \mathrm{C}$; ${ }^{1} \mathrm{HNMR}\left(400 \mathrm{MHz}, \mathrm{CDCl}_{3}\right): \delta=2.13(\mathrm{~s}, 3 \mathrm{H}), 6.44(\mathrm{~s}, 1 \mathrm{H}), 6.96(\mathrm{~d}, J=$ $8.0 \mathrm{~Hz}, 2 \mathrm{H}), 7.39-7.43(\mathrm{~m}, 4 \mathrm{H}), 7.47(\mathrm{~d}, J=8.8 \mathrm{~Hz}, 2 \mathrm{H}), 7.54-7.58(\mathrm{~m}, 2 \mathrm{H}), 7.78-8.39(\mathrm{~m}, 6 \mathrm{H})$; ${ }^{13} \mathrm{CNMR}\left(100 \mathrm{MHz}, \mathrm{CDCl}_{3}\right): \delta=20.8,37.6,117.4,118.0,122.7,124.2,126.7,128.7,129.1,131.4,135.8$, 142.1, 148.7; IR ( $\left.\mathrm{KBr} \mathrm{Cm}^{-1}\right): 3074,1623,1515,1258,1123,1086,966,841,744 ; \mathrm{m} / \mathrm{z}(\%)=372\left(\mathrm{M}^{+}\right)$.

\section{CONCLUSION}

In brief the authors explored a simple and greenery method for one-pot synthesis of 14-aryl-14H dibenzo $[a, j]$ xanthene derivatives by the cyclocondensation of $\beta$-naphthol and aryl aldehydes with biodegradable THC as a bio catalyst under solvent-free and micro wave conditions with several compensation such as high yields, very low reaction times and cost-effective ecofriendly catalyst with simple work-procedure.

\section{ACKNOWLEDGMENT}

We greatly acknowledge to the head of the chemistry department, Osmania University for laboratory facilities and technical support with the manuscript.

\section{REFERENCES}

1. K. Ganesh, C. Nandi, R.K. Varma, M.S. Singh,Tetrahedron Lett.,51, 442 (2010), DOI:10.1016/j.tetlet.2009.11.064

2. M.M. Amini, M. Seyyedhamzeh, A. Bazgir, Applied Catal., A. General, 323, 242(2007), DOI: 10.1016/j.apcata.2007.02.016

3. R.Giri, J. R.Goodell, C. Xing, A. Benoit, H.Kaur, H. Hiasa, D. M.Ferguson, Bioorganic \&Medicinal Chem.,18,1456, (2010), DOI:10.1016/j.bmc.2010.01.018

4. M.A. Pasha, V.P. Jayashankara,Bioorganic \& Medicinal Chem. Lett.,17, 621 (2007), DOI: 10.1016/j.bmcl.2006.11.009

5. A.K. Bhattacharya, K.C. Rana, M. Mujahidinyion,R. Sehar, A.K. Saxena, Bioorganic \& Medicinal Chem, Lett.,19, 5590 (2009), DOI:10.1016/j.bmcl.2009.08.033

6. J. Venu Madhav, Y. Thirupathi Reddy, P. Narsimha Reddy, M. Nikhil Reddy, S. Kuarma, P. A. Crooks , B. Rajitha, J. Mol. Catal.A Chemical, 304, 85 (2009), DOI:10.1016/j.molcata.2009.01.028 
RASĀYAN J. Chem.

Vol. 12 | No. 1 |152 - 156| January - March | 2019

7. B. Rajitha, B.S. Kumar, Y.T. Reddy, P.N. Reddy, N. Sreenivasulu,Tetrahedron Lett.,46, 8691 (2005), DOI: $10.1016 / j$.tetlet.2005.10.057

8. M.M. Amini, M. Seyyedhamzeh, A. Bazgir, Applied Catal. A. General, 323, 242(2007), DOI: 10.1016/j.apcata.2007.02.016

9. M.A. Bigdeli, M.M. Heravi, G. H. Mahdavinia, J. Molecular Catal. A. Chemical,275, 25 (2007), DOI: $10.1016 /$ j.molcata.2007.05.007

10. G. Chen Li, J. Chem, Research, 484, (2008), DOI: 10.3184/030823408X340771

11. H.R. Shaterian, M. Ghashang, J. Braz. Chem. Soc.,19, 1053, (2008), DOI:10.1590/S010350532008000500034

12. H.R. Shaterian, M. Ghashang,N. Mir, ARKIVOC,15, 1, (2007), DOI:10.3998/ark.5550190.0008.f01

13. B.C. Raju, D.V.S. Pradeep, P.P. Reddy, J.M. Rao, Lett. Organic Chem., 5, 450, (2008), DOI: 10.2174/157017808785740435

14. M. Mokhtary, S. Refahati, Dyes and Pigments, 99, 378, (2013), DOI: 10.1016/j.dyepig.2013.05.023

15. F. Shirini,N.G. Khaligh, Dyes and Pigments, 95, 789, (2012), DOI: 10.1016/j.dyepig.2012.06.022

16. J. Ghomi, M.A.Ghasemzadeh, J. Saudi Chem. Soc., 19, 642 (2015), DOI: 10.1016/j.jscs.2012.05.007

17. D. Prasad, A. Preetam, M. Nath, Comptes Rendus Chimie, 15, 675, (2012), DOI: 10.1016/j.crci.2012.05.018

18. H. Naeimi, Z.S. Nazifi, Comptes Rendus Chimie,17, 41, (2014), DOI: 10.1016/j.crci.2013.08.003

19. G.M. Ziarani, A.R. Badiei, M. Azizi, Scientia Iranica,18, 453 (2011), DOI: 10.1016/j.scient.2011.05.008

20. F. Shirini, M.Abedini, R. Pourhasan, Dyes and Pigments,99, 250(2013), DOI: 10.1016/j.dyepig.2013.04.036

21. K. Gong, D. Fang, H.L. Wang, X. Li Zhou, Z.L. Liu. Dyes and Pigments, 80, 30, (2009), DOI: 10.1016/j.dyepig.2008.02.011

22. F. Shirini, N.G. Khaligh, G.H.Imanzadeh, P.G. Ghasem-Abadi, Chinese.Chem.Lett., 23, 1145, (2012), DOI: $10.1016 /$ j.cclet.2012.08.008

23. L. Perreux, A. Loupy, Tetrahedron, 57, 9199, (2001), DOI: 10.1016/S0040-4020(01)00905-X

24. D.R. Baghurst, D.M.P. Mingos, J. Chem. Soc. Chem. Commun., 829, (1988), DOI: 10.1039/C39880000829

25. A. Loupy, Microwaves in Organic Synthesis (Ed.: Wiley-VCH, Weinheim, 2002.

26. R.S. Varma, D.E. Clark, W.H. Sutton, D.A. Lewis, American Ceramic Society, Westerville, Ohio, 357, (1997)

27. P. LidstrUm, J. P. Tierney, Microwave-Assisted Organic Synthesis, Blackwell, Oxford, 2004.

28. C.O. Kappe, Angew. Chem. Int. Ed., 43, 6250 (2004), DOI: 10.1002/anie. 200400655

[RJC-5043/2018] 